\title{
Evolution of primordial neutrino helicities in cosmic gravitational inhomogeneities
}

\author{
Gordon Baym® and Jen-Chieh Peng® \\ Illinois Center for Advanced Studies of the Universe and Department of Physics, \\ University of Illinois, 1110 West Green Street, Urbana, Illinois 61801, USA
}

(Received 19 March 2021; accepted 24 May 2021; published 22 June 2021; corrected 12 November 2021)

\begin{abstract}
Relic neutrinos from the big bang decoupled from the hot plasma predominantly in helicity eigenstates. Their subsequent propagation through gravitational inhomogeneities of the Universe alters the helicities of both Dirac and Majorana neutrinos, thus providing an independent probe of the evolving Universe. We determine here the probability that relic neutrinos flip their helicity, in terms of the spectrum of density inhomogeneities measured in the cosmic microwave background. As we find, for Dirac neutrinos the gravitational helicity modifications are intermediate between the effects of magnetic fields if the neutrino magnetic moment is of the magnitude predicted in the standard model and the much larger effects if the magnetic moment is of the scale consistent with the excess of low energy electron events seen by the XENON1T experiment. We give succinct derivations, within general relativity, of the semiclassical response of a spinning particle to a weak gravitational field in an expanding Universe and estimate the helicity modifications of neutrinos emitted by the Sun caused by the Sun's gravity.
\end{abstract}

DOI: 10.1103/PhysRevD.103.123019

\section{INTRODUCTION}

The cosmic neutrino background $(\mathrm{C} \nu \mathrm{B})$, analogous to the cosmic microwave background (CMB), carries invaluable independent information on the early Universe [1-4]. The primordial electron, muon, and tau neutrinos decoupled in helicity eigenstates at temperatures $\sim \mathrm{MeV}$, much greater than neutrino masses, and cooled in the expanding Universe to a present temperature $\sim 1.7 \times 10^{-4} \mathrm{eV}$. Detection of the $\mathrm{C} \nu \mathrm{B}$, a major experimental challenge, remains an elusive goal. The PTOLEMY experiment [5] proposes to use inverse tritium beta decay (ITBD) [6], $\nu_{\mathrm{e}}+{ }^{3} \mathrm{H} \rightarrow \mathrm{e}^{-}+{ }^{3} \mathrm{He}$, to capture the relic neutrinos. As the ITBD detection rate depends on the helicity as well as the Dirac vs Majorana nature of the relic neutrinos [3,7], a key question is to investigate how the helicity of relic neutrinos evolve as they propagate through the Universe.

As first noted in Ref. [8] a neutrino propagating in a gravitational field can develop an amplitude to have its helicity reversed; as the neutrino trajectory is bent by a gravitational field, the bending of its spin lags the bending of the momentum $[9,10]$. A simple example is a finite mass neutrino with negative helicity shot straight upward from Earth at less than escape velocity; the neutrino will at a

Published by the American Physical Society under the terms of the Creative Commons Attribution 4.0 International license. Further distribution of this work must maintain attribution to the author(s) and the published article's title, journal citation, and DOI. Funded by SCOAP ${ }^{3}$. certain point reverse course and fall back down, but its spin direction will not be affected by the Earth's gravity (neglecting the Lense-Thirring effect from the Earth's rotation). The result is that the neutrino returns with its momentum parallel to its spin, i.e., its helicity is flipped. As another example, the momentum of a nonrelativistic neutrino in a circular orbit around a nonrotating gravitating point mass precesses by angle $2 \pi$ per orbit, while the spin precession is a relativistic correction [11]. Thus nonrelativistically the neutrino helicity oscillates between negative and positive helicity in half an orbit.

A second effect that can modify the helicity of Dirac, but not Majorana, neutrinos arises from their expected magnetic moment [1,12-19], which is diagonal in the mass eigenstate basis. Majorana neutrinos can only have nondiagonal transition magnetic moments between different mass eigenstates. As a Dirac neutrino propagates through astrophysical magnetic fields, from cosmic to galactic to magnetic fields in supernovae and neutron stars, its spin precesses and its helicity is modified. As we discussed, the helicity modification is sensitive both to the neutrino magnetic moment and to the characteristics of the magnetic fields [7]. In estimating the helicity flipping probability for relic neutrinos in both cosmic and galactic magnetic fields, we found that even a neutrino magnetic moment well below the value suggested by the XENON1T experiment could significantly affect the helicities of relic neutrinos and their detection rate via the ITBD reaction [7].

We focus here on the gravitational effect on the helicities of relic neutrinos as they propagate from the time of 
decoupling in the early Universe, of order one second after the big bang, to the present. Owing to the charged current interaction for $\nu_{e}$ and $\bar{\nu}_{e}$, the reaction cross sections for electron neutrinos are larger than for muon and tau neutrinos. An immediate consequence is that electron neutrinos decouple from the plasma of the early Universe at a later time and at a lower temperature than muon and tau neutrinos. As estimated in Ref. [1], $\nu_{\tau}$ and $\nu_{\mu}$ freeze out at temperature $T_{\mu} \sim 1.5 \mathrm{MeV}$, while $\nu_{e}$ freeze out at temperature $T_{e} \sim 1.3 \mathrm{MeV}$. However, the temperature differences at freeze-out do not effect the present temperature, $T_{\nu 0}=1.945 \pm 0.001 \mathrm{~K}=(1.676 \pm 0.001) \times$ $10^{-4} \mathrm{eV}$, of the various neutrino species [a factor $(11 / 4)^{1 / 3}$ smaller than that of the cosmic microwave background].

Relic neutrinos are produced in flavor eigenstates, a coherent sum of neutrino mass eigenstates, and in wave packets whose structure is determined effectively by the electrons and positrons scattering with the $\nu$ and $\bar{\nu}$. The wave packets are limited in size by electron mean free paths at the time of decoupling. As calculated in Ref. [20], a characteristic electron mean free path is of order $1 / \alpha^{2} T$ to within logarithmic corrections, where $\alpha=e^{2} / 4 \pi$; thus at $T \sim 1 \mathrm{MeV}$, the electron mean free path is of order $10^{6}-10^{7} \mathrm{fm}$.

The wave packets of flavor eigenstates quickly disperse into three effectively decoherent wave packets each with a given mass, owing to their velocity differences. The velocity dispersion of the mass eigenstates of a relativistic neutrino with momentum $p$ at decoupling is $\delta v / c \simeq$ $\frac{1}{2} \Delta m^{2} / p^{2}$, where $\Delta m^{2}$ is the characteristic neutrino mass-squared splitting [21]. With $\Delta m^{2}$ on the characteristic scale of $10^{-4} \mathrm{eV}^{2}$, the velocity dispersion for $p \sim 1 \mathrm{MeV}$ is $\sim 1.5 \times 10^{-6} \mathrm{~cm} / \mathrm{sec}$; thus in the first second alone after neutrinos are decoupled, dispersion would spread the mass components some $10^{7} \mathrm{fm}$, at least on the scale of the wave packets in which the neutrinos are produced. The decrease of $p$ in time only increases the velocity dispersion. By contrast, the velocity dispersion within a wave packet of definite mass, $\sim(\delta p / p) m^{2} / p^{2}$, is much smaller, since $\delta p$ within a wave packet is small compared with the packet's mean momentum $p$.

At freeze-out the neutrinos are left in a relativistic thermal distribution,

$$
f(p)=\frac{1}{e^{p / T}+1},
$$

where $p$ is the neutrino momentum and $T$ is the temperature; this distribution is maintained throughout the evolution of the Universe, even though neutrinos in at least two of the three mass states are nonrelativistic at present.

In Sec. II, we lay out the basic physics of momentum spin rotation by a weak gravitational potential, giving selfcontained semiclassical derivations from general relativity of the effects in the Appendix A. Then in Sec. III we calculate the net momentum rotation of primordial neutrinos propagating through the gravitational inhomogeneities of the expanding Universe - the gravitational lensing of the $\mathrm{C} \nu \mathrm{B}$ - and the net helicity changes the neutrinos undergo. As a related application we estimate in Sec. IV the expected helicity rotation of solar neutrinos caused by their gravitational interaction with the Sun itself. In the concluding Sec. V, we compare the gravitational bending with the rotation of neutrino spins owing to a finite neutrino magnetic moment, estimated earlier [7]. Appendix B provides a detailed derivation of the bending of neutrinos emitted from compact spherical objects such as the Sun, neutron stars, and supernovae. We work in units with $\hbar=c=1$.

\section{SPIN ROTATION IN A WEAK GRAVITATIONAL POTENTIAL}

When a particle of mass $m$ and velocity $\vec{v}$ propagates through a weak gravitational potential $\Phi$ its direction of momentum $\hat{p}$ bends at a rate

$$
\left.\frac{d \hat{p}}{d t}\right|_{\perp}=-\left(v+\frac{1}{v}\right) \vec{\nabla}_{\perp} \Phi
$$

where the gradient is taken perpendicular to the direction of momentum. We measure the spin precession in $\Phi$ in terms of the particle spin $\vec{S}$ in the particle's local Lorentz rest frame, reached by a Lorentz boost without rotation. The spin precesses at the slower rate $[9,22]$,

$$
\left.\frac{d \vec{S}}{d t}\right|_{\perp}=-\frac{2 \gamma+1}{\gamma+1} \vec{S} \cdot \vec{v} \vec{\nabla}_{\perp} \Phi
$$

where $\gamma=1 / \sqrt{1-v^{2}}$ is the usual Lorentz factor. These results are derived in Appendix A, including the expansion of the Universe. In a helicity eigenstate $\hat{S} \cdot \hat{p}=\hat{S} \cdot \hat{v}=$ $h= \pm 1$, one has equivalently,

$$
\left[h \frac{d \hat{S}}{d t}-\frac{d \hat{p}}{d t}\right]_{\perp}=\frac{m}{p} \vec{\nabla}_{\perp} \Phi .
$$

As a consequence of the spin lagging the momentum, the helicity of the particle is rotated by gravitational fields. For total angular bend $\delta \theta_{p}$ of the momentum, determined by Eq. (2), the angular bend $\delta \theta$ of the spin with respect to the momentum is thus

$$
\delta \theta=\delta \theta_{s}-\delta \theta_{p}=-\frac{\delta \theta_{p}}{\gamma\left(1+v^{2}\right)},
$$

where $\delta \theta_{s}$ is the bending angle of the spin, calculated from Eq. (3). 


\section{A. Helicity change in passing a distant point mass}

A simple application is the deflection of a relativistic spinning particle passing a distant point mass $M$. Integrating the transverse acceleration (2) over the particle trajectory from $t=-\infty$ to $\infty$ one finds the expected deflection,

$$
\Delta \theta_{p}=\frac{2 M G}{b v^{2}}\left(1+v^{2}\right)
$$

where $G$ is the Newtonian gravitational constant, and $b$ is the impact parameter. (For $v=1$ this is the Einstein weak field light-bending result.) The spin axis precesses by the smaller amount,

$$
\Delta \theta_{s}=\frac{2 M G}{b} \frac{2 \gamma+1}{\gamma+1}
$$

and the angular change of the spin axis with respect to the momentum axis is

$$
\Delta \theta=-\frac{2 M G}{b \gamma v^{2}} .
$$

In the fully relativistic limit, the spin tracks the momentum, leading to no change in the particle helicity. On the other hand, in the nonrelativistic limit the spin rotates negligibly compared with the bending of its momentum, and thus a change in direction of the momentum leads to a change in particle helicity. For spin rotation with respect to the momentum by angle $\theta$ from an initial helicity state, the helicity changes from \pm 1 to $\pm \cos \theta$, and the probability of observing the spin flipped to the opposite direction, which is half the magnitude of the change in helicity, is then $P_{f}=\sin ^{2}(\theta / 2)$.

\section{INTEGRATING OVER THE EXPANSION OF THE UNIVERSE}

We now calculate the momentum bendings and then spin rotations, as neutrinos propagate past the density fluctuations in the early Universe. To take into account the expansion of the Universe, we work in terms of the standard Friedman-Robertson-Walker metric,

$$
d s^{2}=a(u)^{2}\left[-d u^{2}+d \vec{x}^{2}\right] .
$$

Here $u$ is the conformal time, related to coordinate time $t$ by $d t=a(u) d u$, with the metric in homogeneous space, and $\vec{x}$ are the comoving spatial coordinates, related to the usual spatial coordinates $\vec{r}$ by $d \vec{r}=a(u) d \vec{x}$. We take $a(u)=1$ at present.

In the presence of small energy density fluctuations, $\rho(x)=\bar{\rho}+\delta \rho(x)$, with $\bar{\rho}$ the spatially uniform average density, the metric (9) becomes [23]

$$
d s^{2}=a(u)^{2}\left[-(1+2 \Phi) d u^{2}+(1-2 \Phi) d \vec{x}^{2}\right],
$$

where the scalar potential $\Phi$ is given in terms of the density fluctuations by

$$
\nabla_{x}^{2} \Phi=4 \pi G(\delta \rho(\vec{x})+3 \delta P(\vec{x})) a(u)^{2},
$$

with $\delta P$ as the variation of the pressure from uniformity, and $a^{-1} \nabla_{x}$ as the gradient with respect to $\vec{r}$.

In the matter-dominated era (denoted by $\mathcal{M}$ ), the pressure term can be neglected, and (11) becomes the familiar Newtonian equation. Furthermore in this era linear perturbation theory [24] implies that

$$
\delta(\vec{x}) \equiv \delta \rho(\vec{x}) / \bar{\rho}
$$

grows as $a$, where $\bar{\rho}$ is the average density; thus since $\bar{\rho}$ scales as $1 / a^{3}$, we see immediately that $\delta \rho(\vec{x})$ scales as $a^{-2}$ and thus $\nabla_{x}^{2} \Phi(\vec{x})$ and $\Phi(\vec{x})$ as functions of $\vec{x}$ are constant in time.

In the radiation-dominated era (denoted by $\mathcal{R}), \Phi(\vec{x})$ as a function of $x$ is also constant in time, since in this era linear perturbation theory implies that $\delta$ grows rather as $a^{2}$ at large scales, while $\bar{\rho}$ and $\bar{P}$ scale as $1 / a^{4}$. Furthermore the pressure fluctuations in this era are simply $1 / 3$ of the density fluctuations, so that $\nabla_{x}^{2} \Phi=8 \pi G a^{2} \bar{\rho}(x) \delta(\vec{x})$.

To calculate the angular changes in the trajectory of a neutrino, we neglect the neutrino mass at this point for simplicity. Then Eq. (2) gives a total angular change $-2 \int d \ell \nabla_{x \perp} \Phi(\vec{x})$, where $\ell$ is the comoving length along the path. To lowest order the integral is along the straight path of the neutrino, parametrized in the absence of density fluctuations by the coordinate $x_{3}$. The average of the square of the angular deflection of the particle trajectory is then

$$
\left\langle\left(\Delta \theta_{p}\right)^{2}\right\rangle=4 \int d x_{3} d x_{3}^{\prime} \vec{\nabla}_{x \perp} \cdot \vec{\nabla}_{x^{\prime} \perp}\left\langle\Phi\left(x_{3}\right) \Phi\left(x_{3}^{\prime}\right)\right\rangle,
$$

where

$$
\left\langle\Phi(\vec{x}) \Phi\left(\vec{x}^{\prime}\right)\right\rangle=\int \frac{d^{3} k}{(2 \pi)^{3}} e^{i \vec{k} \cdot\left(\vec{x}-\vec{x}^{\prime}\right)} \Psi(k)
$$

is the spatially isotropic, (conformal) time-independent autocorrelation function of the gravitational perturbations; the vectors $\vec{k}$ are comoving.

Then

$$
\left\langle\left(\Delta \theta_{p}\right)^{2}\right\rangle=4 \int d x_{3} d x_{3}^{\prime} \int \frac{d^{3} k}{(2 \pi)^{3}} e^{i k_{3}\left(x_{3}-x_{3}^{\prime}\right)} k_{\perp}^{2} \Psi(k) .
$$

The integration over $x_{3}^{\prime}$ essentially gives $2 \pi \delta\left(k_{3}\right)$, so that 


$$
\left\langle\left(\Delta \theta_{p}\right)^{2}\right\rangle=\frac{2}{\pi} \int d u \int d k_{\perp} k_{\perp}^{3} \Psi\left(k_{\perp}\right)
$$

where $x_{3}=u$ along the trajectory of the neutrino.

The spectral function $\Psi(k)$ is directly related to the spectral function of the density correlation function,

$$
\left\langle\delta(\vec{x}) \delta\left(\vec{x}^{\prime}\right)\right\rangle=\int \frac{d^{3} k}{(2 \pi)^{3}} e^{i \vec{k} \cdot\left(\vec{x}-\vec{x}^{\prime}\right)} P(k),
$$

by

$$
\Psi(k)=\left(4 \pi G \bar{\rho} a^{2}\right)^{2} \zeta \frac{P(k)}{k^{4}},
$$

with $\zeta=1$ in $\mathcal{M}$, and 4 in $\mathcal{R}$ where $\delta P=\delta \rho / 3$.

The spectral function $P(k)$ (with dimensions of volume) depends on the magnitude of $\vec{k}$ and the time. Its general structure [25] is an approximately Harrison-Zel'dovich long wavelength linear growth in $k$ below a maximum at wave vector $k_{H}$; for $k>k_{H}, P(k)$ falls roughly as $k^{-\nu}$ with $\nu>0$. For $k$ below $k_{H}, P(k)$ scales in $\mathcal{M}$ as $a^{2}$ (even beyond the peak at $k_{H}$ ) and as $a^{4}$ in $\mathcal{R}$. In terms of $P(k)$ (with the subscript $\perp$ on the integration variable dropped),

$$
\left\langle\left(\Delta \theta_{p}\right)^{2}\right\rangle=32 \pi \zeta \int d u\left(G \bar{\rho} a^{2}\right)^{2} \int \frac{d k}{k} P(k) .
$$

The angular bending of the neutrino trajectories and modification of the helicity are largest in the matterdominated era, on which we now focus. We include dark energy, which affects the cosmological expansion after redshifts of order $1 / 2$. The relation between the scale factor and the conformal time is determined by

$$
\frac{d a}{d u}=\sqrt{\frac{8 \pi G \bar{\rho}(a) a^{4}}{3}}=H_{0} \sqrt{\Omega_{M} a+\Omega_{V} a^{4}}
$$

where $\bar{\rho}(a)=\rho_{M} / a^{3}+\rho_{V}$, with $\rho_{M} / \rho_{c} \equiv \Omega_{M} \simeq 0.32$ the present average mass fraction (including dark matter) in the Universe, $\rho_{V} / \rho_{c} \equiv \Omega_{V} \simeq 0.68$ the dark energy fraction, and $\rho_{c}$ the present critical closure density; $H_{0}=\sqrt{8 \pi G \rho_{c} / 3}$ is the present Hubble constant [26,27].

With $P_{0}(k)=P(k) / a^{2}$, the angular deviations produced in propagation from matter-radiation equality [where $a\left(t_{e q}\right) \equiv a_{e q} \sim 0.8 \times 10^{-4}$ ] to now are given by

$$
\left\langle\left(\Delta \theta_{p}\right)^{2}\right\rangle \simeq \frac{9}{2 \pi} H_{0}^{4} \mathcal{P} \int_{u_{e q}}^{u_{0}} d u\left(\Omega_{M}+\Omega_{V} a^{3}\right)^{2},
$$

where $\mathcal{P} \equiv \int_{0}^{\infty}(d k / k) P_{0}(k)$. Numerical integration of the Planck Collaboration data ([25] Fig. 19), yields $\mathcal{P} \simeq$ $7.25 \times 10^{4}(\mathrm{Mpc} / \mathrm{h})^{3}$.

Using $a$ as the independent integration variable in evaluating the rotation angles, we find

$$
\left\langle\left(\Delta \theta_{p}\right)^{2}\right\rangle=\frac{9}{2 \pi} \mathcal{P} H_{0}^{3} \int_{a_{e q}}^{1} \frac{d a}{a^{2}}\left(\Omega_{M} a+\Omega_{V} a^{4}\right)^{3 / 2} .
$$

The $a$ integral is approximately 0.56 . In addition $\mathcal{P} H_{0}^{3} \simeq$ $2.69 \times 10^{-6}$ (independent of the Hubble parameter $h$ ), and thus

$$
\left\langle\left(\Delta \theta_{p}\right)^{2}\right\rangle \simeq 2.2 \times 10^{-6} .
$$

This result indicates that gravitational lensing of the CMB would be $\sim 5.1$ arcmin, within a factor of 2 of the value $\sim 2.7$ arcmin from more precise calculations, ${ }^{1}$ e.g., [28].

We now consider the effect of the neutrino mass, which is significant only in $\mathcal{M}$. For finite mass, the integration over $u, \int d u \ldots$, in Eq. (21) now becomes

$$
\frac{1}{4} \int_{u_{e q}}^{u_{0}} d u v(u)\left(v(u)+\frac{1}{v(u)}\right)^{2} \ldots
$$

as one sees from Eq. (2), with $d x_{3}=v(u) d u$. This modification leads to

$$
\begin{aligned}
\left\langle\left(\Delta \theta_{p}\right)^{2}\right\rangle= & \frac{9}{8 \pi} \mathcal{P} H_{0}^{3} \int_{a_{e q}}^{1} \frac{d a}{a^{2}}\left(\Omega_{M} a+\Omega_{V} a^{4}\right)^{3 / 2} \\
& \times v(a)\left(v(a)+\frac{1}{v(a)}\right)^{2} .
\end{aligned}
$$

The velocity of a neutrino of momentum $p_{0}$ at present, and thus with a comoving momentum $p=p_{0} / a$, is $v(a)=1 / \sqrt{1+m_{\nu}^{2} a^{2} / p_{0}^{2}}$. The root-mean-square bending angle $\sqrt{\left\langle\left(\Delta \theta_{p}\right)^{2}\right\rangle}$ is shown in Fig. 1 as a function of the neutrino mass.

In the limit of a very slow neutrino $p_{0} / m_{\nu} \ll 1$, the integral in Eq. (25) is $\simeq 0.3 m_{\nu} / p_{0}$, and we find

$$
\left\langle\left(\Delta \theta_{p}\right)^{2}\right\rangle \simeq \frac{2.7}{8 \pi} \mathcal{P} H_{0}^{3} \frac{m_{\nu}}{p_{0}}
$$

the bending of a nonrelativistic neutrino is larger, as one sees in Fig. 1, than the bending of a relativistic neutrino.

In the radiation-dominated era, from the time of neutrino decoupling, $t_{d} \sim 1 \mathrm{~s}$, to matter-radiation equality, the scale factor is linear in conformal time, $a(u)=\left(8 \pi G \bar{\rho} a^{4} / 3\right)^{1 / 2} u$, and thus from Eq. (19),

\footnotetext{
${ }^{1}$ Owing to reionization of intergalactic $\mathrm{H}$ atoms below redshift $z \sim 10$ and subsequent photon-electron scattering, the lensing of the CMB is most efficient at lower redshift. (Neutrino lensing does not experience such restrictions; the weak electron-neutrino scattering after reionization is insignificant in comparison.) For example, integration over a sharply limited range of $z<6$ in Eq. (22) reduces the mean bending angle to $\sim 3.9$ arcmin.
} 


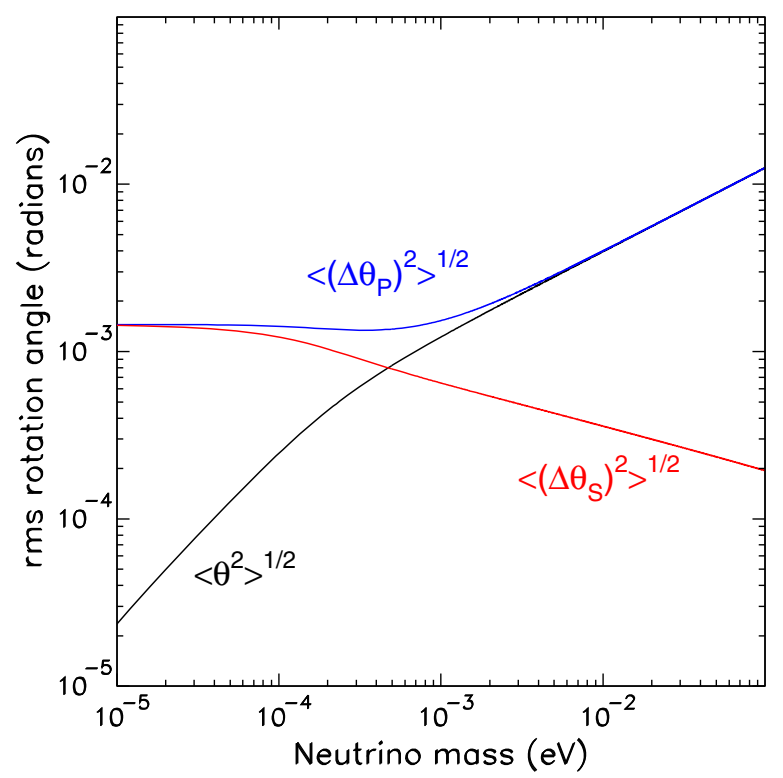

FIG. 1. The root-mean-square bending angles of the neutrino momentum $\sqrt{\left\langle\left(\Delta \theta_{p}\right)^{2}\right\rangle}$, spin $\sqrt{\left\langle\left(\Delta \theta_{s}\right)^{2}\right\rangle}$, and the bending of the spin with respect to the momentum $\sqrt{\left\langle\theta^{2}\right\rangle}$, Eq. (31), in the matter-dominated era, as functions of the neutrino mass. All curves are calculated for the neutrino momentum equal to the present neutrino temperature. The contribution to the bending angles from the radiation-dominated era is negligible.

$$
\left\langle\left(\Delta \theta_{p}\right)^{2}\right\rangle=\frac{18}{\pi} \frac{a_{e q}^{4}}{u_{e q}^{4}} \int_{u_{d}}^{u_{e q}} \frac{d u}{a(u)^{4}} \int \frac{d k}{k} P(k, u) .
$$

Density fluctuations grow in $\mathcal{R}$ as $a^{2}$, and thus $P(k)$ grows as $a^{4}$ outside the horizon scale. The horizon grows as $t \sim a^{2}$ so that the physical wave vector of the horizon decreases as $1 / a^{2}$ and the comoving wave vector decreases as $1 / a$. This implies that the maximum $P\left(k_{H}\right)$ of $P(k)$ for comoving $k$ grows as $a^{3}$, until matter-radiation equilibrium, after which it grows as $a^{2}$. Since $\int d k P(k) / k$ is essentially proportional to $P\left(k_{H}\right)$, we infer

$$
\begin{aligned}
\int \frac{d k}{k} P(k, u) & \simeq \frac{a(u)^{3}}{a_{e q}^{3}} \int \frac{d k}{k} P\left(k, u_{e q}\right) \\
& \simeq \frac{a(u)^{3}}{a_{e q}} \int \frac{d k}{k} P_{0}(k) .
\end{aligned}
$$

With (27),

$$
\begin{aligned}
\left\langle\left(\Delta \theta_{p}\right)^{2}\right\rangle & \simeq \frac{18}{\pi} \frac{a_{e q}^{2}}{u_{e q}^{3}} \ln \left(\frac{a_{e q}}{a_{d}}\right) \int \frac{d k}{k} P_{0}(k), \\
& \sim a_{e q}^{1 / 2} \ln \left(\frac{a_{e q}}{a_{d}}\right) \mathcal{P} H_{0}^{3},
\end{aligned}
$$

where $a\left(u_{d}\right) \equiv a_{d} \sim 2.3 \times 10^{-10}$, and we scale to the present, writing $u_{e q} \sim a_{e q}^{1 / 2} / H_{0}$. The squared angular bending of momentum in the radiation-dominated era is thus of order a few percent of that in the matter-dominated era, Eq. (22).

The spin axis rotates away from the momentum axis only in the matter-dominated regime, where the finite neutrino mass can play a role. To estimate the rotation of the spin itself, we replace according to Eq. (3), the factor $(v+1 / v)$ by $v(2 \gamma+1) /(\gamma+1)$ in Eq. (25), so that

$$
\begin{aligned}
\left\langle\left(\Delta \theta_{s}\right)^{2}\right\rangle= & \frac{9}{8 \pi} \mathcal{P} H_{0}^{3} \int_{0}^{1} \frac{d a}{a^{2}}\left(\Omega_{M} a+\Omega_{V} a^{4}\right)^{3 / 2} \\
& \times v^{3}\left(\frac{2 \gamma+1}{\gamma+1}\right)^{2} .
\end{aligned}
$$

Similarly the probability of spin rotation away from a pure helicity state is, according to Eqs. (2) and (5), given by Eq. (25) with the factor $(v+1 / v)$ by $1 / \gamma v=m_{\nu} / p$,

$$
\left\langle\theta^{2}\right\rangle=\frac{9}{8 \pi} \mathcal{P} H_{0}^{3} \int_{0}^{1} \frac{d a}{a^{2}}\left(\Omega_{M} a+\Omega_{V} a^{4}\right)^{3 / 2}\left(\frac{1}{v}-v\right),
$$

where

$$
\left(\frac{1}{v}-v\right)=\frac{m^{2} a^{2}}{p_{0} \sqrt{p_{0}^{2}+m^{2} a^{2}}} .
$$

Figure 1 shows the bending of the momentum, Eq. (25), the bending of the spin, calculated using Eq. (30), and the bending of the spin axis with respect to the momentum axis, Eq. (31), as a function the mass of the neutrino, for the

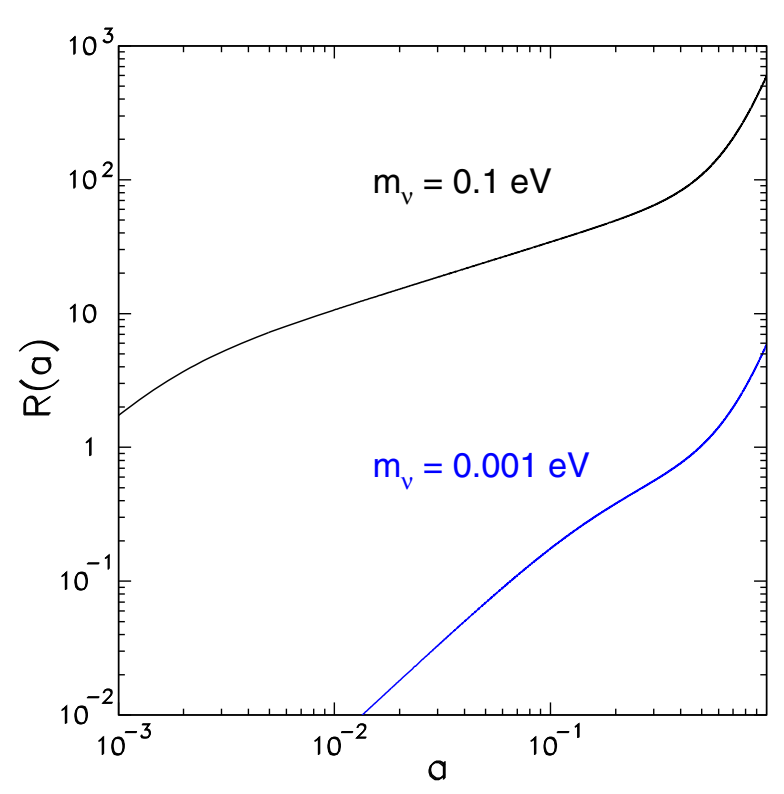

FIG. 2. The integrand $R(a)$ in of the $a$ integral in Eq. (31), showing the dependence of the root-mean-square bending angle of the neutrino spin relative to the momentum as a function of the scale factor $a$, for two neutrino masses, and momentum equal to the present neutrino temperature. 
neutrino momentum equal to the temperature. Similarly Fig. 2 shows the root-mean-square bending angle of the spin with respect to the momentum as a function of the scale factor $a$ for two representative neutrino masses. As this figure shows, the onset of the role of dark energy in the expansion of the Universe leads to a relative increase in the bending in recent epochs, $a \gtrsim 0.3$.

The equality of the spin rotation with respect to the momentum and the momentum rotation for a nonrelativistic neutrino, seen in Fig. 1, is simply a consequence of the absence of spin rotation of a nonrelativistic neutrino in a gravitational field; for a relativistic neutrino, $\left\langle\theta^{2}\right\rangle$ is suppressed by a factor $\left(m_{\nu}^{2} / 2 p_{0}\right)^{2}$ compared with the momentum bending (23). To put the scale of bending in context, we note from Eq. (8) that the spin rotation of a marginally nonrelativistic neutrino $\left(p \sim m_{\nu}\right.$ ) is of order that a neutrino would experience in passing a solar mass neutron star at a distance $\lesssim 10^{4} \mathrm{~km}$.

\section{HELICITY CHANGES OF SOLAR NEUTRINOS}

A related application of helicity rotation by gravitational fields is the spin rotation of solar neutrinos in the gravitational field of the Sun. To estimate the effects, we consider neutrinos emitted in the $z$ direction, focusing first on those emitted at a given transverse distance $b$ from the $z$ axis and distance $r_{0}$ from the center of the star. Since emission at $-b$ leads to the same helicity change as $b$, and there is no coherence between emission from the points $\pm|b|$, we may take $b>0$ throughout. Then the relative bending of the spin and momentum of these neutrinos is, from Eq. (4), given by

$$
\gamma v^{2} \theta\left(b, r_{0}\right)=\int_{z_{0}}^{\infty} d z \nabla_{y} \Phi(r)=-b \int_{z_{0}}^{\infty} d z \frac{G M(r)}{r^{3}},
$$

where $M(r)$ is the stellar mass interior to radius $r$, and $z_{0}= \pm \sqrt{r_{0}^{2}-b^{2}}$, with $z$ measured from the center of the star. The dependence on the neutrino mass is entirely through the velocity-dependent factor $1 / \gamma v^{2}$.

Owing to the spherical symmetry of the Sun, the average bending of the neutrinos beginning at the two values of $z_{0}$ is just the same as if the neutrinos started from $z_{0}=0$. Thus, in calculating the average helicity bending angle, we can replace the lower limit in the integral by 0 ; the average is independent of $r_{0}$. Averaging as well over the solar volume, weighted by $p_{\nu}(r)$, the normalized distribution of neutrino production in the Sun, we derive, as detailed in Appendix B, the average bending angle

$$
\langle\theta\rangle=-\frac{G}{\gamma v^{2}} \int_{0}^{R_{\odot}} 4 \pi r_{0} d r_{0} p_{\nu}\left(r_{0}\right) \int_{0}^{\infty} d r \frac{M(r)}{r^{2}} f\left(r, r_{0}\right),
$$

where

$$
f\left(r, r_{0}\right)=\Theta\left(r_{0}-r\right) r W\left(r_{0} / r\right)+\Theta\left(r-r_{0}\right) r_{0} W\left(r / r_{0}\right)
$$

with the elliptic integral

$$
W(\xi)=\int_{0}^{1} d x \frac{\sqrt{1-x^{2}}}{\sqrt{\xi^{2}-1+x^{2}}}, \quad \xi>1 .
$$

Equation (34) is a convenient starting point for integrating numerically over the empirical mass distribution $M(r)$ and neutrino emissivity distribution $p_{\nu}(r)$ of the Sun; using solar model distributions [29] we find

$$
\langle\theta\rangle=-\frac{1.54}{\gamma v^{2}} \frac{G M}{R} .
$$

For a uniform mass density $\rho(r)$ and uniform $p_{\nu}(r)$, the prefactor becomes 0.76 .

As seen in Fig. 3 the helicity bending angle $|\langle\theta\rangle|$ of nonrelativistic solar neutrinos is sizable; however, only a tiny fraction of solar neutrinos are nonrelativistic. On the other hand, heavy particles with nonzero spin, such as dark photons, emitted from the Sun would have their helicities significantly modified by the Sun's gravitational field. How such a helicity rotation of dark photon could be observed remains an interesting question.

To understand the magnitude of the helicity angle bending from the Sun, we note that the average emission radius of neutrinos $\left\langle r_{0}\right\rangle=\int d^{3} r r p_{\nu}(r)$ is $\simeq 0.11 R_{\odot}$, and thus $b \ll R_{\odot}$. Since $b=r_{0} \sin \omega$, where $\omega$ is the polar angle, the average value of $b$ is $\pi\left\langle r_{0}\right\rangle / 4$. We can thus replace the $z$ integral in Eq. (33) approximately by $\int_{0}^{\infty} d r G M(r) / r^{3}$, independent of $b$; with a simple

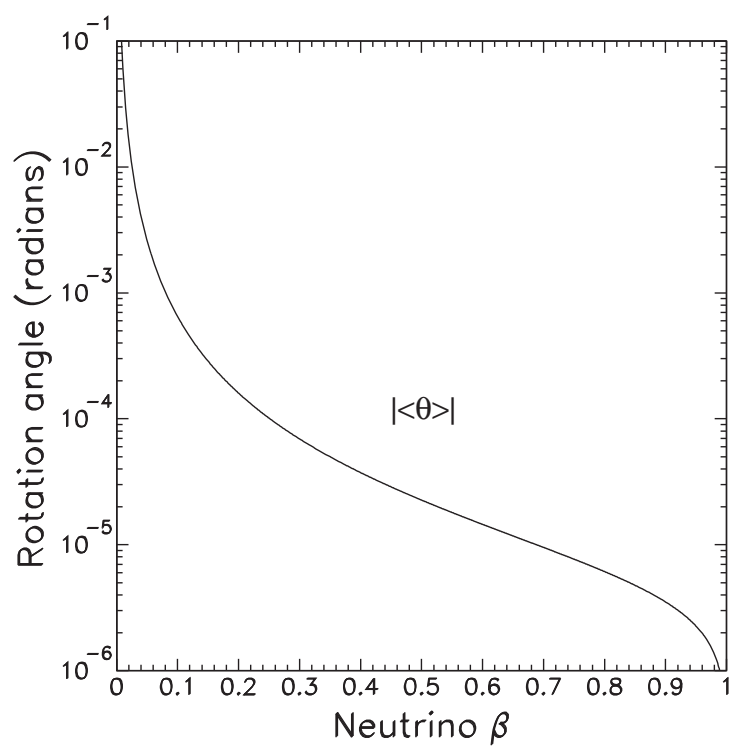

FIG. 3. The mean helicity rotation angle $|\langle\theta\rangle|$ for solar neutrinos as a function of the neutrino $\beta=v / c$. 
integration by parts using $d M(r) / d r=4 \pi \rho(r) r^{2}$, where $\rho(r)$ is the mass density, gives

$$
\langle\theta\rangle \sim-\frac{\pi^{2}\left\langle r_{0}\right\rangle G}{2 \gamma v^{2}} \int_{0}^{\infty} \rho(r) d r .
$$

The density in the Sun falls very approximately as $\rho(r)=\rho_{c}\left(1-r / R^{*}\right)$, where $\rho_{c}$ is the central density, and $R^{*} \sim 0.3 R_{\odot}$. From the solar model [29], $\int d r \rho(r) \simeq$ $3.6 M_{\odot} / R_{\odot}^{2}$, so that

$$
\langle\theta\rangle \sim-\left\{\frac{3 \pi}{16} \frac{\left\langle r_{0}\right\rangle}{R_{\odot}} \frac{R^{*}}{R_{\odot}} \frac{\rho_{c}}{\bar{\rho}}\right\} \frac{G M_{\odot}}{\gamma v^{2} R_{\odot}} \simeq-\frac{2.0}{\gamma v^{2}} \frac{G M_{\odot}}{R_{\odot}},
$$

where $\bar{\rho}$ is the average solar mass density. This estimate is valid to leading order in $b$; the $20 \%$ difference from the numerical result (37) arises from negative corrections of relative order $-2\left(b / R^{*}\right)^{2} \ln \left(R^{*} / b\right)$.

A similar calculation can be carried out for neutrinos emitted from a neutron star or supernova. The characteristic helicity rotation is $\sim G M / \gamma R$, which for $10 \mathrm{MeV}$ scale neutrinos is negligible compared with the magnetic rotation produced even by a neutrino magnetic moment of order estimated in the standard model [7].

\section{IMPLICATIONS}

Gravitational perturbations act equally on Dirac and Majorana neutrinos. As relic left-handed Dirac neutrinos are flipped to right-handed, an equal number of righthanded antineutrinos are flipped to left-handed, and since particles and antiparticles are distinguishable, one could in principle see the depletion experimentally. On the other hand, if neutrinos are Majorana, the reduction in lefthanded neutrinos would not be observable, since the produced left-handed antineutrinos could not be distinguished experimentally from left-handed neutrinos.

An initially negative helicity relic neutrino, after traveling past the gravitational inhomogeneities in the Universe, would have a probability now of being measured with positive helicity, $P_{f}=\left\langle\sin ^{2}(\theta / 2)\right\rangle$. For a presently relativistic neutrino, with mass less than $10^{-4} \mathrm{eV}$, the flipping probability is $\sim 6 \times 10^{-7}$. Since the heaviest neutrino has a mass at least $50 \mathrm{meV}$ [21], scattering from density fluctuations should lead, as one sees from Fig 1, to a population of right-handed relic neutrinos and left-handed relic antineutrinos approaching one in $10^{5}$. This effect is too small to be seen in planned experiments to detect relic neutrinos [3,5] via inverse tritium decay reaction [6], but it is not beyond the range of eventual measurability.

Earlier [7], we estimated that the bending of the spin of a Dirac neutrino with a diagonal magnetic moment $\mu_{\nu}$, as it travels through a galaxy, is of order

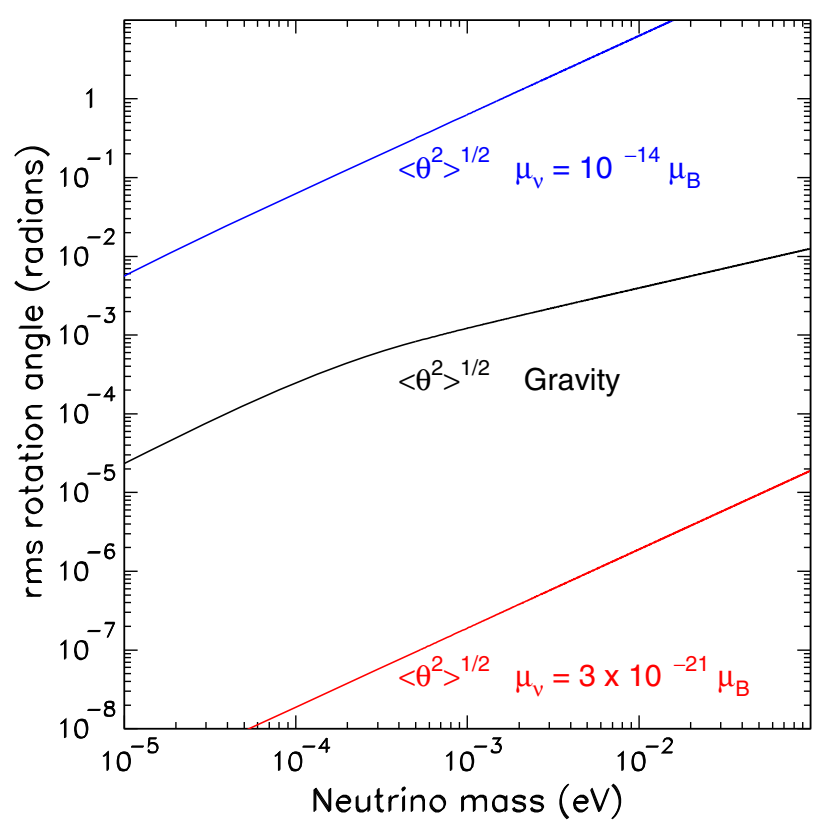

FIG. 4. Comparison of the root-mean-square bending angle $\sqrt{\left\langle\theta^{2}\right\rangle}$ of the spin of a primordial neutrino with respect to its momentum from gravitational vs magnetic effects, as a function of the neutrino mass. All curves are calculated for the neutrino momentum equal to the temperature. The middle curve shows the results of Eq. (31) for the gravitational bending, for both a Dirac and a Majorana neutrino. The upper and lower curves are the bending expected from the interaction of a Dirac neutrino magnetic moment $\mu_{\nu}$, with a characteristic galactic magnetic field $\sim 10 \mu \mathrm{G}$, for the standard model estimate [13] of $\mu_{\nu}$ (lower curve) with $m_{\nu}=10^{-2} \mathrm{eV}$, and for a magnetic moment $10^{-14} \mu_{B}$, 3 orders of magnitude below that which would explain the XENON1T low energy electron events [30] (upper curve).

$$
\left\langle\theta^{2}\right\rangle_{g} \simeq\left(\frac{\mu_{\nu} B_{g}}{v}\right)^{2} \ell_{g} \Lambda_{g}
$$

where $B$ is the average galactic magnetic field, $\ell_{g}$ is a mean crossing distance of the galaxy, $\Lambda_{g}$ is the characteristic coherence length of the field, and $\mu_{B}$ is the Bohr magneton. Unlike gravitational spin bending, the spins of Majorana neutrinos would not be affected by magnetic fields since Majorana neutrinos can have only transition magnetic moments, and the interactions with slowly varying astrophysical magnetic fields cannot change the neutrino mass.

Equations (26) and (40) indicate that the scale of spin bending of a nonrelativistic thermal neutrino of mass $m_{\nu}=$ $10^{-2} \mathrm{eV}$ by density fluctuations is comparable to that produced by a galactic magnetic field $\sim 10 \mu \mathrm{G}$, with $\Lambda_{g} \sim 1$ and $\ell_{g} \sim 16 \mathrm{kpc}$, if the neutrino has a magnetic moment $\mu_{\nu} \sim 5 \times 10^{-18}$. As we see in Fig. 4 , the scale of gravitational bending of a neutrino spin with respect to its momentum is well above the magnetic bending produced by the standard model estimate of the magnetic moment [12-14], $\sim 3 \times 10^{-21} m_{-2} \mu_{B}$, where $m_{-2}$ is the neutrino 
mass in units of $10^{-2} \mathrm{eV}$, but well below that produced by a magnetic moment $1.4-2.9 \times 10^{-11} \mu_{B}$ that would explain the excess of low energy electron events in the XENON1T experiment [30]. See discussion in Ref. [7].

Quite generally, neutrino helicity modification, although not measurable by current experiment, is a potentially important probe of cosmic gravitational fields, as well as the interiors of compact objects including the Sun, neutron stars, and supernovae.

\section{ACKNOWLEDGMENTS}

This research was supported in part by NSF Grant No. PHY18-22502. We thank Jessie Shelton, Gil Holder, Stu Shapiro, and Michael Turner for helpful discussions.

\section{APPENDIX A: BENDING OF MOMENTA AND SPINS IN WEAK GRAVITATIONAL FIELDS}

In this Appendix we summarize the derivations of Eqs. (2) and (3) for the bending of the momentum and spin in a weak gravitational potential, including the expansion of the Universe in the metric, Eq. (10).

The equation of motion of a particle with proper velocity $U^{\mu} \equiv d x^{\mu} / d \tau$, where $\tau$ is the proper time of the particle, propagating through a general gravitational field, is given by the geodesic equation,

$$
\frac{d U^{\mu}}{d \tau}+\Gamma_{\alpha \beta}^{\mu} U^{\alpha} U^{\beta}=0,
$$

where $\Gamma_{\alpha \beta}^{\mu}=\frac{1}{2} g^{\mu \nu}\left(\partial_{\beta} g_{\nu \alpha}+\partial_{\alpha} g_{\nu \beta}-\partial_{\nu} g_{\alpha \beta}\right)$ is the affine connection. Using the explicit components of the affine connection for the metric $(10),{ }^{2}$ we see that the spatial velocity $\vec{U}^{i}$ obeys

$$
\begin{aligned}
\frac{d U^{i}}{d \tau}= & -\nabla_{i} \Phi\left(\left(U^{0}\right)^{2}+(\vec{U})^{2}\right)+2 U^{i}(\vec{U} \cdot \vec{\nabla}) \Phi \\
& -\frac{2}{a} \frac{d a}{d \tau} U^{i} U^{0} .
\end{aligned}
$$

For acceleration along $\vec{U}$, the second term on the first line changes the $\left(U^{0}\right)^{2}+(\vec{U})^{2}$ to $\left(U^{0}\right)^{2}-(\vec{U})^{2}$ which equals $1 / a^{2}$ to zeroth order in $\Phi$; thus $d\left(a^{2} U^{i}\right) / d \tau=-\nabla^{i} \Phi$ along $\vec{U}$.

The four-momentum $p_{\mu}=m g_{\mu \nu} U^{\nu}$ in general obeys

$$
\begin{aligned}
\frac{d p_{\mu}}{d \tau} & =m \frac{d g_{\mu \nu}}{d \tau} U^{\nu}+m g_{\mu \nu} \frac{d U^{\nu}}{d \tau} \\
& =\frac{m}{2}\left(\partial_{\mu} g_{\alpha \beta}\right) U^{\alpha} U^{\beta},
\end{aligned}
$$

\footnotetext{
${ }^{2}$ The nonvanishing components of the affine connection are $\Gamma_{00}^{i}=\Gamma_{i 0}^{0}=\nabla_{i} \Phi, \quad \Gamma_{j k}^{i}=-\nabla_{k} \Phi \delta_{j}^{i}-\nabla_{j} \Phi \delta_{k}^{i}+\nabla_{i} \Phi \delta^{j} k, \quad \Gamma_{00}^{0}=$ $a^{-1} d a / d x^{0}, \quad \Gamma_{j 0}^{i}=\Gamma_{0 j}^{i}=\delta_{j}^{i} a^{-1} d a / d x^{0}, \quad$ and $\quad \Gamma_{i j}^{0}=\delta_{i j}(1-$ $4 \Phi) a^{-1} d a / d x^{0}$.
}

where to find the second line we use $d A / d \tau=U^{\mu} d A / d x^{\mu}$, for a function $A$, as well as the geodesic equation combined with the definition of the affine connection. In the weak field metric with expansion (10), the spatial momentum $p_{i}$ thus obeys

$\frac{d p_{i}}{d \tau}=\frac{m}{2}\left(\partial_{i} g_{\alpha \beta}\right) U^{\alpha} U^{\beta}=-m a^{2} \nabla_{i} \Phi\left(\left(U^{0}\right)^{2}+\left(U^{j}\right)^{2}\right)$.

Since $d t / d \tau=\gamma$ to zeroth order in $\Phi$, we find, with expansion,

$$
\frac{1}{|\vec{p}|} \frac{d \vec{p}}{d t}=-\left(\frac{1}{v}+v\right) \vec{\nabla} \Phi
$$

where $\vec{v}=d \vec{x} / d u$. Equation (2) follows immediately. Similarly, in the metric (10) (by definition, $p_{0}<0$ ),

$$
\frac{d p_{0}}{d \tau}=\frac{m}{2}\left(\partial_{0} g_{\alpha \beta}\right) U^{\alpha} U^{\beta}=-\frac{m}{a} \frac{\partial a}{\partial x^{0}},
$$

since $g_{\alpha \beta} U^{\alpha} U^{\beta}=-1$. Thus $p_{0} a$ is conserved.

We turn now to spin precession. ${ }^{3}$ The helicity is defined in terms of the spin $\vec{S}$ in the local Lorentz frame at rest with respect to the particle. In this frame $S^{0} \equiv 0$. To determine the equation of motion for $\vec{S}$, we begin with the spin $\tilde{S}^{\mu}$ in the local Lorentz frame at rest in the "lab," which obeys the normalization condition, $\tilde{S}_{\mu} \tilde{S}^{\mu}=\vec{S}^{2}$, and relate $\tilde{S}^{\mu}$ to the spin in the weak field metric, denoted here by $\Sigma^{\mu}$.

The normalization condition on $\Sigma^{\mu}$ is

$$
\Sigma_{\mu} \Sigma^{\mu}=-a^{2}(1+2 \Phi)\left(\Sigma^{0}\right)^{2}+a^{2}(1-2 \Phi) \vec{\Sigma}^{2}=\vec{S}^{2}
$$

Thus to first order in $\Phi$,

$$
\tilde{S}^{i}=a(1-\Phi) \Sigma^{i}, \quad \tilde{S}^{0}=a(1+\Phi) \Sigma^{0} .
$$

In addition, $\Sigma_{\mu} U^{\mu}=0$, to guarantee that the spin in the particle rest frame has no time component.

The particle spin in the weak field metric obeys the geodesic equation

$$
\frac{d \Sigma^{\mu}}{d \tau}+\Gamma_{\alpha \beta}^{\mu} \Sigma^{\alpha} U^{\beta}=0
$$

and thus

\footnotetext{
${ }^{3}$ The spin motion was earlier analyzed for a general static metric in Ref. [22] in terms of the tetrad formalism and for a Dirac particle in Ref. [9] using a Foldy-Wouthuysen transformation of the Dirac equation.
} 


$$
\begin{aligned}
\frac{d \vec{\Sigma}}{d \tau}= & -2 \vec{\nabla} \Phi(\vec{\Sigma} \cdot \vec{U})+(\vec{U} \cdot \vec{\nabla} \Phi) \vec{\Sigma}+(\vec{\Sigma} \cdot \vec{\nabla} \Phi) \vec{U} \\
& -\frac{1}{a} \frac{d a}{d x^{0}}\left(U^{0} \vec{\Sigma}+\Sigma^{0} \vec{U}\right) .
\end{aligned}
$$

Equation (A8) implies that to order $\Phi$ the component of the equation of motion of $\overrightarrow{\tilde{S}}$ transverse to $\vec{U}$ obeys

$$
\left.\frac{d \overrightarrow{\tilde{S}}}{d \tau}\right|_{\perp}=\left.\frac{d}{d \tau}(a(1-\Phi) \vec{\Sigma})\right|_{\perp}=-2 \vec{\nabla}_{\perp} \Phi(\overrightarrow{\tilde{S}} \cdot \vec{U}) .
$$

Equivalently, $d \overrightarrow{\tilde{S}} /\left.d t\right|_{\perp}=-2 \overrightarrow{\nabla_{\perp}} \Phi(\overrightarrow{\tilde{S}} \cdot \vec{v})$, which combined with Eq. (A5) shows that, for a massless particle, the spin direction in the lab Lorentz frame remains parallel (or antiparallel) to the momentum.

At this stage we transform back to the local Lorentz frame at rest with respect to the particle. Since $S^{0} \equiv 0$, the spins in the two Lorentz frames are related by

$$
\overrightarrow{\tilde{S}}=\vec{S}+(\tilde{\gamma}-1) \hat{v}(\hat{v} \cdot \vec{S}),
$$

where $\tilde{\gamma}=\left(1-\tilde{v}^{2}\right)^{-1 / 2}$, with the velocity difference of the two Lorentz frames given by $\overrightarrow{\tilde{v}}=[(1+\Phi) /(1-\Phi)] \vec{v}$. In components parallel and perpendicular to $\vec{v}, \tilde{S}_{\perp}=S_{\perp}$, and $\tilde{S}_{\|}=\gamma S_{\|}$. Thus

$$
\left.\frac{d \vec{S}}{d \tau}\right|_{\perp}-\left.\frac{d \overrightarrow{\tilde{S}}}{d \tau}\right|_{\perp}=-\left.(\tilde{\gamma}-1)(\hat{v} \cdot \vec{S}) \frac{d \hat{v}}{d \tau}\right|_{\perp} .
$$

Since $d \hat{v} / d \tau$ is first order in $\Phi$, we can neglect the distinction between $\overrightarrow{\tilde{v}}$ and $\vec{v}$, and find

$$
\begin{aligned}
\left.\frac{d \vec{S}}{d \tau}\right|_{\perp}-\left.\frac{d \overrightarrow{\tilde{S}}}{d \tau}\right|_{\perp} & =-\left.\frac{\vec{S} \cdot \vec{U}}{(\gamma+1)} \frac{d \vec{U}}{d \tau}\right|_{\perp} \\
& =\frac{1}{\gamma+1}\left(\vec{S} \times\left(\vec{U} \times \frac{d \vec{U}}{d \tau}\right)\right)_{\perp}
\end{aligned}
$$

The latter term is simply the Thomas precession, at lab frequency $\omega_{\mathrm{Th}}=\left(\gamma^{2} /(\gamma+1)\right) \vec{v} \times \dot{\vec{v}}$, of an accelerated particle. With Eqs. (A11) and (A2) we then find

$$
\left.\frac{d \vec{S}}{d \tau}\right|_{\perp}=-\frac{2 \gamma+1}{\gamma+1}(\vec{S} \cdot \vec{U}) \vec{\nabla}_{\perp} \Phi,
$$

from which Eq. (3) follows.

Equivalently,

$$
\left.\frac{d \vec{S}}{d t}\right|_{\perp}=\left.\frac{2 \gamma+1}{\gamma+1}(\vec{S} \times(\vec{v} \times \vec{\nabla} \Phi))\right|_{\perp}
$$

indicating that the spin feels an effective velocity-dependent torque $(\mu \vec{B})_{\text {eff }}=[(2 \gamma+1) / 2(\gamma+1)](\vec{v} \times \vec{\nabla} \Phi)$. The nonrelativistic limit of this equation gives Schiff's result for precession of a spin in the Gravity Probe B experiment [11] (see also Ref. [31]), while in the fully relativistic limit, $\gamma \rightarrow \infty$, the spin remains at the same angle with respect to the momentum.

\section{APPENDIX B: GRAVITATIONAL SPIN ROTATION OF NEUTRINOS EMITTED FROM A SPHERICAL BODY}

We detail here the calculation of the relative spin rotation of neutrinos emitted from a spherical star, applicable to solar neutrinos as well as neutrinos from supernovae and neutron stars. We first convert the $z$ integral in Eq. (33), with $z_{0}$ set to 0 , to an integral over $r$, so that

$$
\gamma v^{2} \theta(b)=-b \int_{b}^{\infty} d r \frac{G M(r)}{r^{2} \sqrt{r^{2}-b^{2}}} .
$$

Then we average the neutrino emission over the stellar volume with a spherically symmetric normalized spatial emission probability $p_{\nu}\left(r_{0}\right) d^{3} r_{0}$, in terms of cylindrical coordinates $\left(d^{3} r_{0}=2 \pi b d b d z\right)$,

$$
\begin{aligned}
\langle\theta(b)\rangle & =\int 2 \pi b d b d z \int d r_{0} p_{\nu}\left(r_{0}\right) \delta\left(r_{0}-\sqrt{b^{2}+z^{2}}\right) \theta(b), \\
& =\int_{0}^{R_{\odot}} 4 \pi r_{0} d r_{0} p_{\nu}\left(r_{0}\right) \int_{0}^{r_{0}} \frac{b d b}{\sqrt{r_{0}^{2}-b^{2}}} \theta(b), \quad \text { (B2) }
\end{aligned}
$$

where in the first line the ranges of the $b$ and $z$ integrals are constrained by the delta function. Thus

$$
\begin{aligned}
\gamma v^{2}\langle\theta\rangle= & -\int_{0}^{R_{\odot}} 4 \pi r_{0} d r_{0} p_{\nu}\left(r_{0}\right) \int_{0}^{r_{0}} \frac{b^{2} d b}{\sqrt{r_{0}^{2}-b^{2}}} \\
& \times \int_{b}^{\infty} d r \frac{G M(r)}{r^{2} \sqrt{r^{2}-b^{2}}} .
\end{aligned}
$$

Interchanging the order of the $r$ and $b$ integrals, we see that their product is equivalent to

$$
\int_{0}^{\infty} d r \frac{G M(r)}{r^{2}} f\left(r, r_{0}\right),
$$

where

$$
f\left(r, r_{0}\right)=\Theta\left(r_{0}-r\right) r W\left(r_{0} / r\right)+\Theta\left(r-r_{0}\right) r_{0} W\left(r / r_{0}\right)
$$

with

$$
\begin{aligned}
W(\xi) & =\int_{0}^{1} \frac{x^{2} d x}{\sqrt{1-x^{2}} \sqrt{\xi^{2}-x^{2}}} \\
& =\int_{0}^{1} d x \frac{\sqrt{1-x^{2}}}{\sqrt{\xi^{2}-1+x^{2}}}, \quad \xi>1 .
\end{aligned}
$$

Equation (34) follows directly. 
[1] A. D. Dolgov, Neutrinos in cosmology, Phys. Rep. 370, 333 (2002).

[2] C. Quigg, Cosmic neutrinos, in Lecture at 2007 SLAC Summer School, arXiv:0802.0013.

[3] A. J. Long, C. Lunardini, and E. Sabancilar, Detecting nonrelativistic cosmic neutrinos by capture on tritium: Phenomenology and physics potential, J. Cosmol. Astropart. Phys. 08 (2014) 038.

[4] J. Y.-Y. Lin and G. Holder, Gravitational lensing of the cosmic neutrino background, J. Cosmol. Astropart. Phys. 04 (2020) 054.

[5] S. Betts et al., Development of a relic neutrino detection experiment at PTOLEMY: Princeton Tritium Observatory for Light, Early-Universe, Massive-Neutrino Yield (Community Summer Study 2013: Snowmass on the Mississippi), arXiv: 1307.4738; E. Baracchini et al., PTOLEMY: A proposal for thermal relic detection of massive neutrinos and directional detection of MeV dark matter; arXiv:1808.01892.

[6] S. Weinberg, Universal neutrino degeneracy, Phys. Rev. 128, 1457 (1962).

[7] G. Baym and J.C. Peng, The Evolution of Primordial Neutrino Helicities in Astrophysical Magnetic Fields and Implications for their Detection, Phys. Rev. Lett. 126, 191803 (2021).

[8] G. Duda, G. Gelmini, and S. Nussinov, Expected signals in relic neutrino detectors, Phys. Rev. D 64, 122001 (2001).

[9] A. J. Silenko and O. V. Teryaev, Semiclassical limit for Dirac particles interacting with a gravitational field, Phys. Rev. D 71, 064016 (2005).

[10] M. Dvornikov, Neutrino spin oscillations in gravitational fields, Int. J. Mod. Phys. D 15, 1017 (2006); Spin effects in neutrino gravitational scattering, Phys. Rev. D 101, 056018 (2020); J. Cosmol. Astropart. Phys. 04 (2021) 005.

[11] L. Schiff, Motion of a gyroscope according to Einstein's theory of gravitation, Proc. Natl. Acad. Sci. U.S.A. 46, 871 (1960).

[12] W. J. Marciano and A. I. Sanda, Exotic decays of the muon and heavy leptons in gauge theories, Phys. Lett. 67B, 303 (1977).

[13] K. Fujikawa and R. Shrock, The Magnetic Moment of a Massive Neutrino and Neutrino Spin Rotation, Phys. Rev. Lett. 45, 963 (1980).

[14] B. W. Lee and R. E. Shrock, Natural suppression of symmetry violation in gauge theories: Muon- and electron-leptonnumber nonconservation, Phys. Rev. D 16, 1444 (1977).

[15] B. W. Lynn, Magnetic moment of massive neutrinos and the cosmic helium abundances, Phys. Rev. D 23, 2151 (1981).

[16] S. L. Shapiro and I. Wasserman, Massive neutrinos, helium production and the primordial magnetic field, Nature (London) 289, 657 (1981).
[17] N. F. Bell, V. Cirigliano, M. J. Ramsey-Musolf, P. Vogel, and M. B. Wise, How Magnetic is the Dirac Neutrino, Phys. Rev. Lett. 95, 151802 (2005).

[18] N. F. Bell, M. Gorchtein, M. J. Ramsey-Musolf, P. Vogel, and P. Wang, Model independent bounds on magnetic moments of Majorana neutrinos, Phys. Lett. B 642, 377 (2006).

[19] C. Giunti and A. Studenikin, Neutrino electromagnetic interactions: A window to new physics, Rev. Mod. Phys. 87, 531 (2015).

[20] H. Heiselberg and G. Baym, The electrical conductivity of early universe, Phys. Rev. D 56, 5254 (1997).

[21] The best-value fits to the mass-squared differences, $\Delta m_{i j}^{2} \equiv m_{i}^{2}-m_{j}^{2}$, are [32] $\Delta m_{21}^{2}=(7.50+0.19-0.17) \times$ $10^{-5}, \Delta m_{31, N}^{2}=(2.524+0.039-0.040) \times 10^{-3}, \Delta m_{31, I}^{2}=$ $(-2.514+0.038-0.041) \times 10^{-3} \mathrm{eV}^{2}$.

[22] N. A. Voronov, Spin precession in a gravitational field, $\mathrm{Zh}$. Eksp. Teor. Fiz. 94, 1 (1988) [Sov. Phys. JETP 67, 2392 (1988)], http://jetp.ac.ru/cgi-bin/dn/e_067_12_2393.pdf.

[23] J. B. Hartle, Gravity, an Introduction to Einstein's General Relativity (Addison-Wesley, San Francisco, 2003).

[24] S. Dodelson, Modern Cosmology (Academic Press, Burlington, MA, 2003).

[25] N. Aghanim et al. (Planck Collaboration), Planck 2018 results I. Overview and the cosmological legacy of Planck, Astron. Astrophys. 641, A1 (2020); see Fig. 19.

[26] J. Frieman, M. Turner, and D. Hurturer, Dark energy and the accelerating Universe, Annu. Rev. Astron. Astrophys. 46, 385 (2008).

[27] N. Aghanim et al. (Planck Collaboration), Planck 2018 results, VI. Cosmological parameters, Astron. Astrophys. 641, A6 (2020).

[28] A. Lewis and A. Challinor, Weak gravitational lensing of the CMB, Phys. Rep. 429, 1 (2006).

[29] J. N. Bahcall and R. K. Ulrich, Solar models, neutrino experiments, and helioseismology, Rev. Mod. Phys. 60, 297 (1988).

[30] E. Aprile et al. (XENON1T Collaboration), Excess electronic recoil events in XENON1T, Phys. Rev. D 102, 072004 (2020).

[31] S. Weinberg, Gravitation and Cosmology: Principles and Applications of the General Theory of Relativity (John Wiley \& Sons, New York, 1972).

[32] I. Esteban, M. C. Gonzalez-Garcia, M. Maltoni, I. MartinezSoler, and T. Schwetz, Updated fit to three neutrino mixing: Exploring the accelerator-reactor complementarity, J. High Energy Phys. 01 (2017) 087.

Correction: Minor errors in Eqs. (A2), (A4), (A10), (A11), and (A13) have been fixed. 